\title{
Perfusion imaging with 320-slice spiral computed tomography and color-coded digital subtraction angiography for assessing acute skeletal muscle ischemia- reperfusion injury in a rabbit model
}

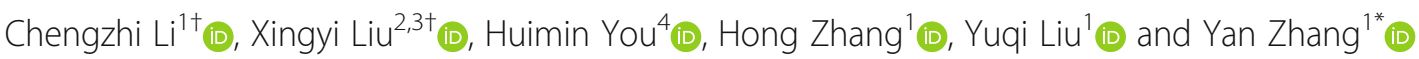

\begin{abstract}
Background: In recent years, skeletal muscle-related ischemia-reperfusion injury (IRI) has become more common. IRI can lead to severe limb injuries, multiple organ failure, and even death in some cases. However, there is still a lack of rapid and sensitive detection methods for IRI in skeletal muscle. This study aims to explore the value of computed tomography perfusion imaging (CTPI) and color-coded digital subtraction angiography (DSA) in assessing acute IRI of skeletal muscle in a rabbit model.
\end{abstract}

Methods: Fifty New Zealand white rabbits were randomly assigned to the ischemia-reperfusion (IR) group $(n=40)$ or sham group $(n=10)$. After $3 \mathrm{~h}$ of surgically-induced hindlimb ischemia, the IR group underwent reperfusion and CTPI and color-coded DSA were taken to assess the skeletal muscle at $0,6,12$, or $24 \mathrm{~h}$ post-reperfusion. The data from CTPI and DSA in the right and left hindlimbs, blood flow (AF-R/L), blood volume (BV-R/L), contrast clearance rate (C-R/L) and the maximum contrast enhancement values (peak-R/L) were obtained. Serum superoxide dismutase (SOD), creatine kinase $(\mathrm{CK})$, lactic dehydrogenase $(\mathrm{LDH})$ and malondialdehyde (MDA) were measured. The statistical correlation between the above parameters (CTPI, color-coded DSA, and biochemical markers) was analyzed.

Results: The mean value of AF-R/L, BV-R/L, C-R/L and peak-R/L decreased linearly from $1.07 \pm 0.08$ to $0.75 \pm 0.11,1.03 \pm$ 0.06 to $0.85 \pm 0.14,0.93 \pm 0.15$ to $0.71 \pm 0.18$, and $1.07 \pm 0.01$ to $0.47 \pm 0.04$, respectively. The correlation coefficients between AF-R/L and SOD, CK, LDH and MDA were $0.57,-0.44,-0.60$, and -0.62 , respectively $(p<0.001)$. The correlation coefficients between Peak-R/L and SOD, CK, LDH, MDA were $0.59,0.68,0.71$ and 0.66 , respectively ( $p<$ 0.001). The correlation coefficient between AF-R/L and Peak-R/L was $0.70(p<0.001)$.

Conclusion: Both CTPI and color-coded DSA could dynamically assess skeletal muscle IRI in rabbits.

Keywords: Ischemia-reperfusion injury, CT perfusion imaging, Color-coded DSA, Skeletal muscle

\footnotetext{
* Correspondence: dsazy@163.com

${ }^{+}$Chengzhi Li and Xingyi Liu are the first two authors contributed equally to this study and should be qualified as co-first authors.

${ }^{1}$ Department of Interventional Radiology and Vascular Surgery, The First Affiliated Hospital of Jinan University, No.613 of West Huangpu Avenue, Guangzhou 510630, China

Full list of author information is available at the end of the article
}

(c) The Author(s). 2019 Open Access This article is distributed under the terms of the Creative Commons Attribution 4.0 International License (http://creativecommons.org/licenses/by/4.0/), which permits unrestricted use, distribution, and reproduction in any medium, provided you give appropriate credit to the original author(s) and the source, provide a link to the Creative Commons license, and indicate if changes were made. The Creative Commons Public Domain Dedication waiver (http://creativecommons.org/publicdomain/zero/1.0/) applies to the data made available in this article, unless otherwise stated. 


\section{Background}

Ischemia-reperfusion injury (IRI) is tissue damage that occurs when the blood supply is restored to tissues after a period of ischemia [1]. The restoration of the blood flow to these tissues leads to microvascular injury, oxidative stress, and inflammation [2]. In recent years, IRI involving skeletal muscle has become more common and can lead to severe limb injuries, multiple organ failure, and even death in some cases [3].

IRI can be evaluated by its clinical manifestations, including clinical symptoms, medical imaging, and measurement of certain serum biochemical biomarkers. Currently, the primary biomarkers evaluated in cases of IRI include creatine kinase (CK), lactate dehydrogenase (LDH), malondialdehyde (MDA), and hydrogen peroxide dismutase (SOD) [4]. While providing vital insight into disease severity, the measurement of these biomarkers requires time and may not be feasible during urgent traumas. For this reason, medical imaging had become a key player in the diagnosis and treatment planning of IRI conditions.

In terms of medical imaging, computed tomography perfusion imaging (CTPI), magnetic resonance imaging (MRI), and digital subtraction angiography (DSA) have been widely used for evaluating IRI in myocardium and brain tissue [5-8]. CTPI is one of the most widely used techniques for detecting and evaluating perfusion in tissues or organs [9]. The advantages of CTPI include its widespread availability, short acquisition times, and simple calculation for perfusion quantification. However, the high radiation exposure has limited the development of CTPI in some cases $[10,11]$. CTPI is used to dynamically scan preselected regions of interest (ROI) within tissues or organs after injection of a contrast agent. After scanning, the time-density curve (TDC) of each pixel in the ROI is obtained. Based on the TDC, blood flow (BF), blood volume (BV), mean moving time of the contrast agent (MMT), and capillary permeability can be calculated using certain mathematical models, thus providing insight into the perfusion state [12].

Color-coded DSA, which is a post-processing technology used to analyze image density changes caused injected contrast agents, converts scan information into continuous-change pixels. The creation of a time-density curve of the bolus injection of the contrast agent in the user-defined regions allows for the calculation of functional parameters like blood-flow distribution and blood-flow velocity $[13,14]$.

CTPI and color-coded DSA have seldom been used to assess IRI in previous studies $[14,15]$. In this study, the combination of 320-slice spiral CTPI and color-coded DSA values were used to evaluate IRI in a rabbit model of hindlimb, to provide guidance and a basis for the effective assessment of acute IRI in clinical practice. As of now, this is the first report comparing the use CTPI and color-coded DSA for evaluating IRI. The advantages and correlations between these two imaging techniques in the evaluation of IRI in skeletal muscle are discussed.

\section{Methods}

\section{Experimental groups}

Fifty New Zealand white rabbits (male, $2.0-2.5 \mathrm{~kg}$ ) were obtained from the Huadong Xinhua Laboratory Animal Center (Guangzhou, China), and randomly assigned to the IR $(n=40)$ or control group $(n=10)$. All rabbits were raised at the Animal Laboratory Center of Jinan University, and housed with five mice per cage. After $3 \mathrm{~h}$ of right hindlimb ischemia, the IR group were assessed at $0,6,12$, or $24 \mathrm{~h}(\mathrm{n}=10$, for each time point) after reperfusion. A sham operation was performed to rabbits in the control group. For all of the IR groups, CTPI (CTPI subgroup, $n=5$ ) and color-coded DSA (DSA subgroup, $n=5$ ) were used to image the bilateral limbs of each animal, respectively.

\section{Creation of the IRI model in rabbits}

The rabbits were anesthetized with an intraperitoneal injection of $1 \%$ pentobarbital sodium $(40 \mathrm{mg} / \mathrm{kg}$ of body weight). Using $1 \%$ pentobarbital sodium $(30 \mathrm{mg} / \mathrm{kg}$ of body weight), subsequent anesthesia was maintained every $3 \mathrm{~h}$ through the ear-rim auricular vein injection. In the IR groups, an anterior abdominal wall incision was made after sterilization. The right iliolumbar artery, right internal iliac artery and right common iliac artery were exposed and ligated with a 3-0 silk suture (Jinhuan, Shanghai, China). The blood supply of the tail was blocked using a tight elastic band to prevent the collaterals to the hindlimb during the ischemia. After confirming successful ischemia induction, the abdomen was closed. At $3 \mathrm{~h}$ post-surgery, the abdomen was reopened and the ligatures were removed. The red color of vessels with a visible pulsation indicated that the reperfusion was successful. In the control group, the abdomen of rabbits was only opened and closed without ligation of the arteries. A flowchart of this study is shown as Fig. 1.

\section{CT scan procedures}

In the CTPI subgroup of IR, the Aquilion One 320 volumetric CT scanner (Toshiba, Japan) was used to scan the bilateral hindlimbs of the experimental rabbits at 0 , 6,12 , or $24 \mathrm{~h}$ post-reperfusion depending on the group (CT-0, CT-6, CT-12, or CT-24, respectively). After sufficient anesthesia, the rabbits were fixed at the center of the CT scanner in a supine position. The scan range included the full-length of the hindlimbs. Before scanning, $3 \mathrm{~mL}$ of iodixanol $(32 \mathrm{mg} / \mathrm{mL})$ was injected with a highpressure syringe at the rate of $1 \mathrm{~mL} / \mathrm{s}$. Following the iodixanol, $8 \mathrm{~mL}$ of physiological saline was injected at 


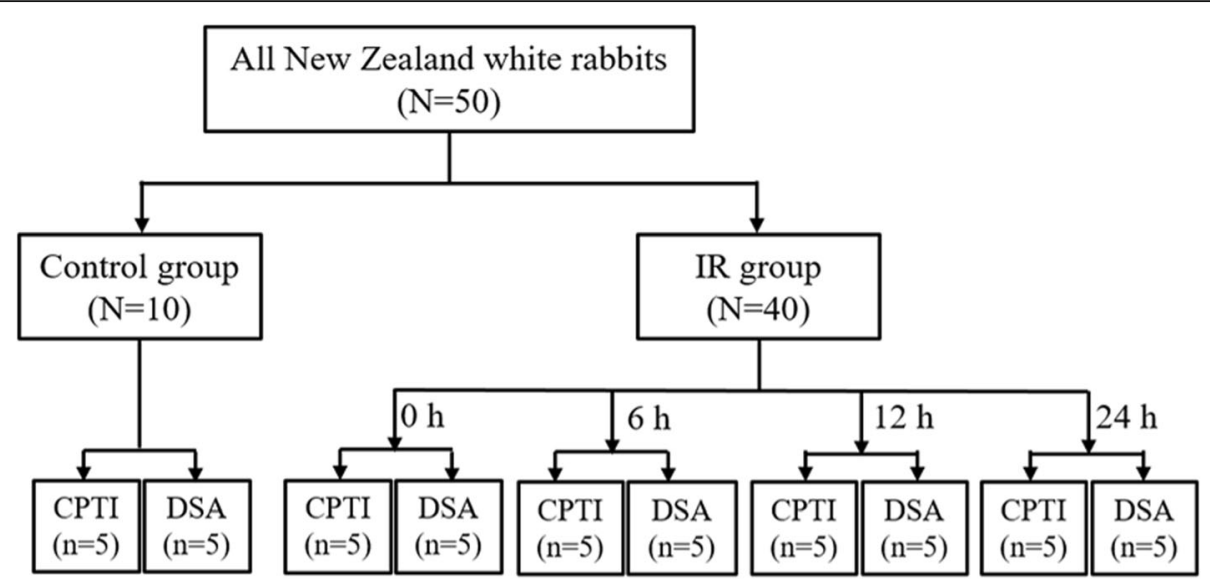

Fig. 1 Flowchart of the present study. The time points indicate the time post-reperfusion. IR, ischemia-reperfusion; CPTI group, computed tomography perfusion imaging; DSA group, color-coded digital subtraction angiography

the same rate through the ear vein. Then $3 \mathrm{~s}$ later, the delayed $\mathrm{x}$-ray scanning was performed. The data collection time was $60.5 \mathrm{~s}$ which included 3 parts. In the initial 22.5 $\mathrm{s}$, data was collected every $1.5 \mathrm{~s}$, and the exposure time was $0.5 \mathrm{~s}$. The collection was performed 12 times during this procedure. In the middle $18 \mathrm{~s}$, data was collected every $2.5 \mathrm{~s}$, and the exposure time was $0.5 \mathrm{~s}$. The collection was performed six times during this procedure. In the last $20 \mathrm{~s}$, data was collected every $4.5 \mathrm{~s}$, and the exposure time was $0.5 \mathrm{~s}$. The collection was performed four times during this procedure. The scanning parameters consisted of the detector arranged by $320 \times 0.5 \mathrm{~mm}$ with a thickness of 0.5 $\mathrm{mm}, 0.5 \mathrm{~s} / \mathrm{ring}, 80 \mathrm{kV}$, and $40 \mathrm{~mA}$.

\section{Angiography procedures}

Similar to the CTPI subgroup, rabbits in the DSA subgroup were fixed onto the operation table in supine position. After supplemental anesthesia and skin preparation, the right main carotid artery was exposed and punctured. Depending on the group (IR-0, IR-6, IR-12, or IR-24), angiography of the lower abdominal aorta was performed to assess changes in the blood flow changes of the right hindlimb using a $2.7 \mathrm{~F}$ microcatheter (Terumo, Japan) through the carotid artery at $0,6,12$, or $24 \mathrm{~h}$. The DSA equipment used in this study was Artis Zeego (Siemens, Erlangen, Germany). Iodixanol $(320 \mathrm{mg} / \mathrm{mL})$ was injected with a velocity of $3 \mathrm{~mL} / \mathrm{s}$ through the microcatheter using a power injector. The injection pressure was $200 \mathrm{psi}$ and the total dose of the contrast agent was $9 \mathrm{~mL}$. The DSA acquisition frame rate was six frames per sec and each acquisition process lasted until the inferior vena cava was visualized.

\section{Image post-processing}

The imaging data collected from CT and DSA were sent to their respective post-processing workstations, respectively (CT: Vitrea, Toshiba, Japan; DSA: Leonardo, Siemens, Germany). The CTPI and color-coded DSA images were automatically generated by the software. Based on the images, the $50-\mathrm{mm}$ region of interest (ROI) was selected in the bilateral vastus lateralis muscle from all the rabbits by two practicing interventional radiologists independently. The ROI was chosen in the soft tissue, yet away from the skeleton and vessels in both the CTPI and DSA groups.

Using the post-processing workstation, perfusion parameters, including blood flow (AF), blood volume $(\mathrm{BV})$, and contrast agent clearance $(\mathrm{C})$ values of the right and left limb ROI (AF-R, AF-L; BV-R, BV-L; C$\mathrm{R}, \mathrm{C}-\mathrm{L}$, respectively) in the CTPI subgroup and the maximum contrast enhancement (peak) of the right and left limb ROI (peak-R, peak-L, respectively) in the DSA subgroup were obtained. To eliminate individual differences among the rabbits in this study, the ratios of $\mathrm{AF}, \mathrm{BV}, \mathrm{C}$, and the peak were calculated during the post-processing (AF-R/L, BV-R/L, C-R/L and peak-R/L).

\section{Testing of blood samples}

Prior to the CT and DSA scanning in each rabbit, $3 \mathrm{~mL}$ of blood was taken from the external jugular vein. After centrifugation of the blood samples, the separated serum was stored at $-20^{\circ} \mathrm{C}$. The samples were used to measure SOD, CK, LDH and MDA. CK and LDH using an automatic biochemical analyzer. In addition, MDA was measured using the thialbarbital sodium method, while SOD was measured via the xanthine oxidase method.

\section{Animal euthanasia}

After the experiment, all rabbits were sacrificed by injecting $10 \mathrm{~mL}$ of air through the ear-rim auricular vein. 


\section{Statistical methods}

SPSS 16.0 (IBM, Chicago, IL, USA) was used for the statistical analysis. A single factor variance analysis was used for comparison between groups. The independentsamples $t$-test was used for comparison between data obtained from the control group and IR group. The correlation of $A F-R / L, B V-R / L, C-R / L$ and peak-R/L between various biochemical indicators, including $S O D$, CK, LDH and MDA, were observed by the Pearson's correlation analysis. The statistical data are expressed as standard error of the mean and $p$-values $<0.05$ were considered significant.

\section{Results}

\section{Establishment of the IRI model in rabbits}

The surgical procedure was successful in all 45 rabbits (Fig. 2). CTPI and color-coded DSA were successfully achieved in each group (Figs. 3 and 4). There were no deaths during this study.

\section{CTPI and color-coded DSA parameters}

The detailed CTPI and color-coded DSA parameters are shown in Tables 1 and 2. For CTPI, a significant difference existed between each experimental group and the control group in AF-R/L $(p<0.001)$. When compared with the control group, significant differences only existed in the $12 \mathrm{~h}$ and $24 \mathrm{~h}$ groups in BV$\mathrm{R} / \mathrm{L}$ and $\mathrm{C}-\mathrm{R} / \mathrm{L}(p<0.001)$. Using a single factor variance analysis, significant differences in AF-R/L were detected between each group when compared with the control group $(\mathrm{F}=6.206, p=0.002)$. The AF-R/L value gradually decreased over time, yet this decrease was not significant between $0 \mathrm{~h}$ and $24 \mathrm{~h}$. The BV-R/L and $\mathrm{C}-\mathrm{R} / \mathrm{L}$ from each group showed no significant differences between each other. For the color-coded DSA, there was a significant difference in Peak-R/L between the control group and all IR groups $(p<$ $0.001)$. Extension of IRI time resulted in a gradual decrease of the Peak-R/L.

\section{Serum biomarkers for assessment of IRI}

As shown in Table 3, SOD in the IR group was lower than in the control group. However, CK, LDH and MDA in the IR groups were higher than those of the sham group $(p<0.001)$. The variance analysis showed that the differences in $\mathrm{CK}$ and $\mathrm{LDH}$ among all groups were statistically significant $(p<0.001)$. CK and LDH increased gradually with the IR time. However, there were no significant differences in MDA or SOD among the groups $(p>0.05)$.

\section{Correlation between indicators: CTPI and biochemical parameters}

In the present study, AF-R/L was positively correlated with SOD $(r=0.57, p<0.001)$ and negatively correlated with $\mathrm{CK}, \mathrm{LDH}$, and MDA $(\mathrm{r}=-0.60, p<0.001 ; \mathrm{r}=-$ $0.44, p<0.001 ; \mathrm{r}=-0.62, P<0.001$; respectively). $\mathrm{LDH}$ was negatively correlated with $\mathrm{BV}-\mathrm{R} / \mathrm{L}(\mathrm{r}=-0.45, p<$

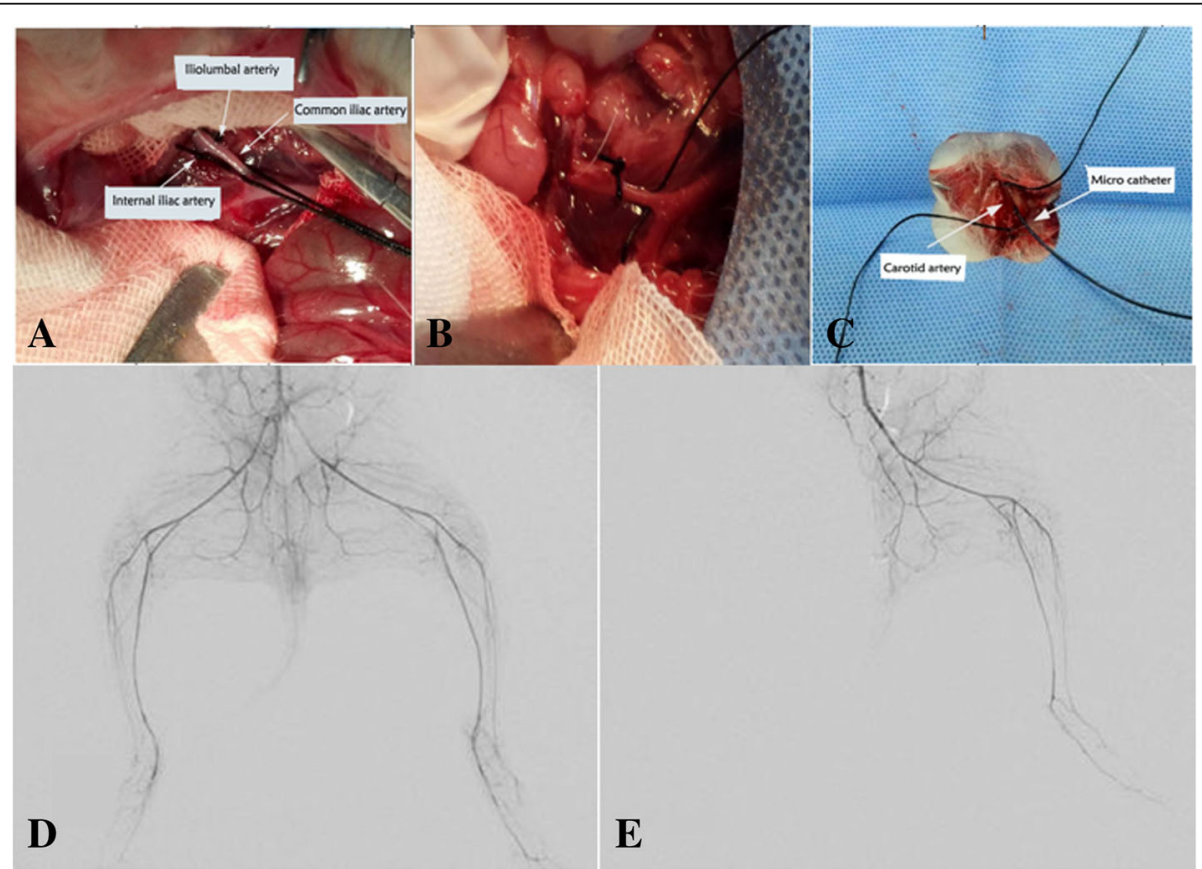

Fig. 2 Establishment of the IRI model in rabbits was accomplished by (a) separating the iliolumbar artery and iliac artery, (b) ligating the iliolumbar artery and iliac artery, (c) performing a unilateral carotid artery catheterization to make the angiogram, (d) angiography of the bilateral hindlimbs confirmed the non-ischemia status, (e) angiography of the bilateral hindlimbs confirmed the ischemia status 


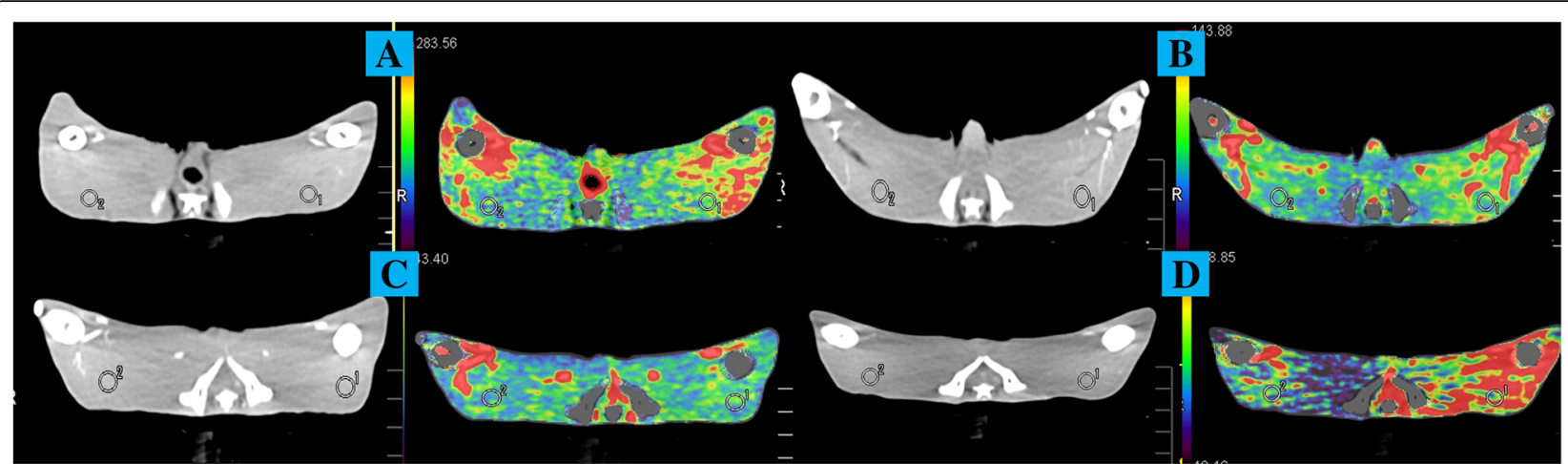

Fig. 3 Plain $C T$ scan and CT perfusion images in the (a) control group, (b) IR-6 h group, (c) IR-12 $\mathrm{h}$ group, and (d) IR-24 $\mathrm{h}$ group

0.001 ), and no correlation existed between any of the biochemical biomarkers and C-R/L.

The Peak-R/L was positively correlated with SOD $(\mathrm{r}=0.59, p<0.001)$ but negatively correlated with $\mathrm{CK}$, LDH and MDA $(\mathrm{r}=-0.68, p<0.001 ; \mathrm{r}=-0.71, p<$ $0.001 ; \mathrm{r}=-0.66, p<0.001$; respectively). The Peak- $\mathrm{R} / \mathrm{L}$ was positively correlated with AF-R/L in the colorcoded DSA and CTPI $(r=0.70, p<0.001)$.

\section{Discussion}

In the present research, the usefulness of CTPI and color-coded DSA for evaluating IRI was investigated using a hindlimb ischemia rabbit model. CTPI and DSA images were obtained from each rabbit in the
CTPI and DSA subgroups at different time points $(0$, 6,12 , or $24 \mathrm{~h}$ after reperfusion). IRI-related serum biochemical biomarkers were measured and the AF$\mathrm{R} / \mathrm{L}, \mathrm{BV}-\mathrm{R} / \mathrm{L}, \mathrm{C}-\mathrm{R} / \mathrm{L}$ of $\mathrm{CT}$ perfusion and the peak-R/ Ls of angiograms were determined and compared among the groups.

A series of studies have shown that CTPI is effective for assessing ISI of the myocardium, brain, and liver. These studies demonstrated that the CTPI parameters of the corresponding tissues and organs changed significantly with of reperfusion injury [16, 17]. In terms of skeletal muscle applications, CTPI can quantitatively evaluate blood flow changes during muscle tissue reperfusion in the hindlimbs of animals

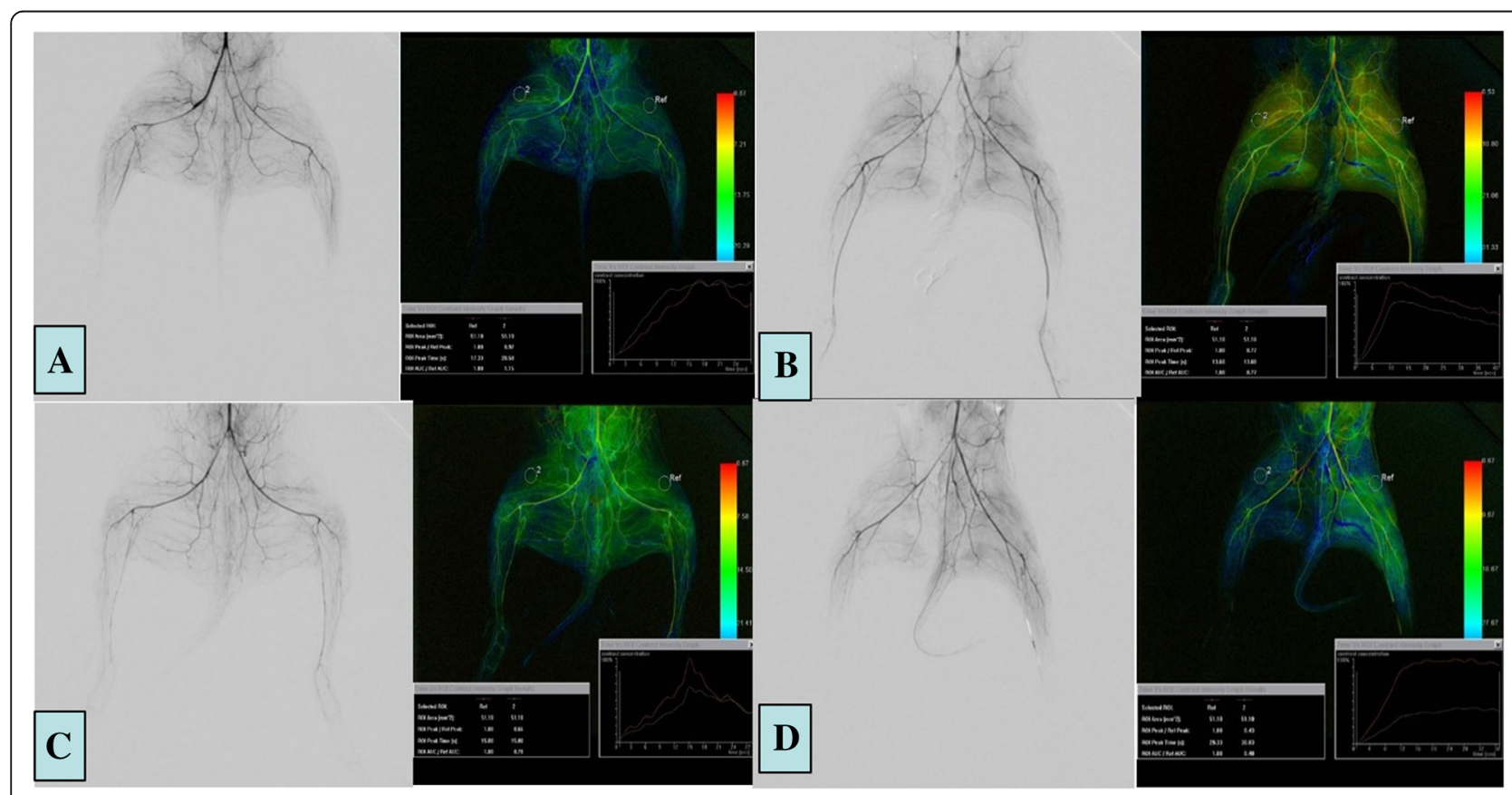

Fig. 4 Color coded DSA post-processing images in the (a) control group, (b) IR-6 h group, (c) IR-12 h group, and (d) IR-24 h group 
Table 1 CTPI parameters for each IR group and the control group $(X \pm S D)$

\begin{tabular}{llll}
\hline Group & AF-R/L & BV-R/L & C-R/L \\
\hline Control & $1.07 \pm 0.08$ & $1.03 \pm 0.06$ & $0.93 \pm 0.15$ \\
IR 0 h & $0.92 \pm 0.14^{*}$ & $0.98 \pm 0.18$ & $0.83 \pm 0.16$ \\
IR 6 h & $0.89 \pm 0.12^{*}$ & $0.97 \pm 0.29$ & $0.79 \pm 0.08$ \\
IR 12 h & $0.88 \pm 0.20^{*}$ & $0.88 \pm 0.11^{*}$ & $0.73 \pm 0.06^{*}$ \\
IR 24 h & $0.75 \pm 0.11^{*}$ & $0.85 \pm 0.14^{*}$ & $0.71 \pm 0.18$ \\
\hline
\end{tabular}

* $p<0.001$ compared to the control group. IR ischemia-reperfusion, $A F$ blood flow, $B V$ blood volume, $C$ contrast agent clearance

with acute or chronic ischemia [18]. However, there are few studies on the application of CTPI in the assessment of IRI. Our data showed that the value of AF-R/L decreased by $30 \%$ by $24 \mathrm{~h}$ with an obvious downward trend after IRI. Moreover, a significant correlation between AF-R/L and serum biochemical indices was observed. These findings indicate that the AF-R/L can better reflect IRI status and assessing AF$\mathrm{R} / \mathrm{L}$ in clinical work may provide additional insight into the extent of damage to skeletal muscle tissues during IRI. Hence, CPTI may be useful in the assessment of IRI.

Color-coded DSA is a kind of imaging technology implemented by post-imaging processing software. Since most studies on color-coded DSA have focused on vascular lesions, most of the literature has used the vascular lumen as the ROI. The peak time of contrast agents in the ROI was measured as a comparative parameter to evaluate hemodynamic changes [19]. While in this study, skeletal muscle IRI was the core of the research, therefore the muscle tissue was considered as the ROI. It was previously shown that IRI in muscle tissue gradually increased in the soleus muscle of rats within $24 \mathrm{~h}$ after $4 \mathrm{~h}$ of ischemia, suggesting that the most serious complications of IRI occurred within $24 \mathrm{~h}$ after reperfusion [5]. Hence, changes that occurred during $0-24 \mathrm{~h}$ postreperfusion were investigated in the present study. Our data showed that the peak-R/Ls gradually

Table 2 Peak-R/L measurements for each IR group and the control group $(X \pm S D)$

\begin{tabular}{ll}
\hline Group & PEAK-R/L \\
\hline Control & $1.09 \pm 0.04$ \\
IR 0 h & $0.93 \pm 0.04^{*}$ \\
IR 6 h & $0.80 \pm 0.06^{*}$ \\
IR 12 h & $0.65 \pm 0.03^{*}$ \\
IR 24 h & $0.45 \pm 0.08^{*}$ \\
\hline${ }^{*} p<0.001$ compared to the control group. IR ischemian
\end{tabular}

${ }^{*} p<0.001$ compared to the control group. IR ischemia-reperfusion, PEAK maximum contrast enhancement decreased during the reperfusion procedure and was the lowest at $24 \mathrm{~h}$ post-reperfusion. Therefore, peak$\mathrm{R} / \mathrm{L}$ can reflect the severity of reperfusion injury to a certain extent, indicating color-coded DSA can be used to evaluate IRI.

Laboratory measurements of SOD, CK, LDH and MDA can also be used to objectively evaluate the extent and severity of IRI [6, 20-22]. This study also investigated the relationship between these biochemical parameters and IRI. The results showed serum levels of CK and LDH were higher in the IRI when compared with the control group. Moreover, these parameters positively correlated with the duration of reperfusion, which suggests that rabbit limbs were damaged by the reperfusion and the damage gradually increased over time. Similarly, MDA gradually increased with the duration of reperfusion, while SOD decreased over time after reperfusion. SOD and MDA indirectly reflected the content of free oxygen radicals, and the increase of MDA and decrease of SOD represents an increased production of free oxygen radicals [23]. Thus, our data indicate that oxidative injury of tissues gradually increases with the duration of IRI.

Our findings suggest that both AF-R/L from CTPI and peak-R/L from color-coded DSA can be used to objectively access IRI. In clinical work, CTPI and color-coded DSA have respective advantages in evaluating IRI. CTPI is a non-invasive approach that is more widely accepted by patients and physicians. Also, CTPI data are derived from sliced images, which increases its accuracy, especially in terms of AF-R/L. However, the patient is exposed to ionizing radiation via the CT scan and contrast agent [10]. Despite the invasiveness of DSA, it has been considered the gold standard for detecting vascular diseases [24]. Compared with CT, DSA requires less time and can be completed during an operation, which can aid surgeons in the identification of disease severity. Furthermore, color-coded DSA can be generated from a conventional DSA sequence with no additional x-ray dose or contrast medium [14]. Our findings suggest that both AF-R/L from CTPI and peak-R/L from color-coded DSA were indicators of IRI, and revealed that IRI worsened with increased time to reperfusion. Moreover, they were positively correlated and also related with some serum biomarkers of IRI. Thus, CPTI and color-coded DSA can be chosen according to the actual status of patients in clinical application.

However, there are several limitations to our study. The imaging changes observed during reperfusion cannot be distinguished from those changes caused by the ischemic injury. Therefore, further comparative studies assessing these two forms of injury, including 
Table 3 Measurements of serum biochemical biomarkers from each IR group and the control group $(X \pm S D)$

\begin{tabular}{lllll}
\hline Group & SOD $(\mathrm{U} / \mathrm{mL})$ & CK $(\mathrm{U} / \mathrm{L})$ & LDH $(\mathrm{U} / \mathrm{L})$ & $\mathrm{MDA}(\mathrm{nmol} / \mathrm{mL})$ \\
\hline Control & $172.82 \pm 7.06$ & $389.75 \pm 42.25$ & $402.38 \pm 26.23$ & $1.51 \pm 0.30$ \\
IR 0 h & $152.14 \pm 15.06^{*}$ & $688.98 \pm 35.22^{*}$ & $568.65 \pm 42.85^{*}$ & $2.03 \pm 0.24^{*}$ \\
IR 6 h & $148.72 \pm 15.80^{*}$ & $3668.19 \pm 599.56^{*}$ & $1172.59 \pm 89.86^{*}$ & $2.11 \pm 0.33^{*}$ \\
IR $12 \mathrm{~h}$ & $142.24 \pm 12.73^{*}$ & $5780.22 \pm 421.64^{*}$ & $1410.76 \pm 198.32^{*}$ & $2.38 \pm 0.46^{*}$ \\
IR $24 \mathrm{~h}$ & $136.12 \pm 11.40^{*}$ & $8954.13 \pm 894.36^{*}$ & $2031.80 \pm 265.28^{*}$ & $3.07 \pm 0.60^{*}$ \\
\hline
\end{tabular}

${ }^{*} p<0.001$ compared to the control group. $I R$ ischemia-reperfusion, $S O D$ hydrogen peroxide dismutase, $C K$ creatine kinase, $L D H$ lactic dehydrogenase, MDA malondialdehyde

reperfusion and ischemia-induced injury, should be conducted in the future.

\section{Conclusions}

In conclusion, the combination of 320-slice spiral CT perfusion analysis and color-coded DSA allows for the dynamic detection and assessment of disease occurrence and severity in rabbits with skeletal muscle IRI.

\section{Abbreviations}

CK: Creatine kinase; CTPI: Computed tomography perfusion imaging; DSA: Digital subtraction angiography; IR: Ischemia-reperfusion; IRI: Ischemiareperfusion injury; LDH: Lactic dehydrogenase; MDA: Malondialdehyde; MRI: Magnetic resonance imaging; SOD: Superoxide dismutase

\section{Acknowledgements}

Not applicable.

\section{Authors' contributions}

$\mathrm{CL}$ designed the study, analyzed and interpreted the experimental data, he was a major contributor in writing the manuscript. $\mathrm{XL}$ carried out the experimental operation. HY collected the experimental data and made the statistical analysis. $\mathrm{HZ}$ carried out the experimental operation. YL carried out the experimental operation and participated in writing the manuscript. YZ designed the study, reviewed and decided to submit manuscript. All authors read and approved the final manuscript.

\section{Funding}

This study was supported by "the Fundamental Research Funds for Central Universities" in China (No.21617318). And it only provided funds for this study without any impact on the research.

\section{Availability of data and materials}

The datasets used and/or analyzed during the current study are available from the corresponding author on reasonable request.

\section{Ethics approval}

The experimental procedures in this study were complied with the $\mathrm{NIH}$ (National Institutes of Health publication 86-23, 1985) Guidelines for Use of Laboratory Animals. This study was also approved by the Animal Ethics Committee of the First Affiliated Hospital of Jinan University.

\section{Consent for publication}

Not applicable.

\section{Competing interests}

The authors declare that they have no competing interests.

\section{Author details}

'Department of Interventional Radiology and Vascular Surgery, The First Affiliated Hospital of Jinan University, No.613 of West Huangpu Avenue, Guangzhou 510630, China. ${ }^{2}$ Graduate School, Guangzhou University of Chinese Medicine, Guangzhou, China. ${ }^{3}$ Second work unit: Department of Interventional Radiology and Vascular Surgery, The First Affiliated Hospital of
Jinan University, No.613 of West Huangpu Avenue, Guangzhou 510630

China. ${ }^{4}$ Department of endocrinology, The Fifth Affiliated Hospital of

Guangzhou medical university, Guangzhou 510700, China.

Received: 3 March 2019 Accepted: 23 June 2019

Published online: 28 August 2019

\section{References}

1. McCord JM. Oxygen-derived free radicals in postischemic tissue injury. New Engl J Med. 1985;312(3):159-63.

2. Huang T, Wang W, Tu C, Yang Z, Bramwell D, Sun X. Hydrogen-rich saline attenuates ischemia reperfusion injury in skeletal muscle. J Surg Res. 2015; 194(2):471-80

3. Duran A, Ciloglu S, Sehirli O, et al. Apocynin and dimethyl sulfoxide synergistically protect against ischemia-reperfusion injury in a rat hind limb ischemia-reperfusion model. EUR J Plast Surg. 2017:40(6):507-12.

4. Bosco G, Yang Z, Nandi J, et al. Effects of hyperbaric oxygen on glucose, lactate, glycerol and anti-oxidant enzymes in the skeletal muscle of rats during ischemia and reperfusion. Clin Exp Pharmacol P. 2007;34(1-2):70-6.

5. Burk LM, Wang KH, Wait JM, et al. Delayed Contrast Enhancement Imaging of a Murine Model for Ischemia Reperfusion with Carbon Nanotube MicroCT. Plos One. 2015:10(1):e0115607.

6. Jiang CJ, Wang ZJ, Zhao YJ, et al. Erythropoietin reduces apoptosis of brain tissue cells in rats after cerebral ischemia/reperfusion injury: a characteristic analysis using magnetic resonance imaging. N R R. 2016;11(9):1450-5.

7. Wang J, Cheng JJ, Huang KY, et al. Quantitative assessment of angiographic perfusion reduction using color-coded digital subtraction angiography during transarterial chemoembolization. Abdom Radiol (NY). 2016;41(3):54552

8. Su H, Lou W, Gu J. Clinical values of hemodynamics assessment by parametric color coding of digital subtraction angiography before and after endovascular therapy for critical limb ischemia. Chinese Med J. 2015;95(37): 3036-40.

9. Elmaadawy MM, Elsorougy LG, Razek AAA, Soliman M, Soliman NY, et al. Perfusion CT: A biomarker for soft tissue tumors of extremities. Egyptian Journal of Radiology \& Nuclear Medicine. 2013;44(4):805-15.

10. Tawfik AM, Razek AA, Elhawary G, Batouty NM. Effect of increasing the sampling interval to 2 seconds on the radiation dose and accuracy of CT perfusion of the head and neck. J Comput Assist Tomogr. 2014;38:469-73.

11. Abdel Razek AA, Denewer AT, Hegazy MA, Hafez MT. Role of computed tomography angiography in the diagnosis of vascular stenosis in head and neck microvascular free flap reconstruction. Int J Oral Maxillofac Surg. 2014; 43:811-5.

12. Koenig M, Kraus M, Theek C, Klotz E, Gehlen W, et al. Quantitative assessment of the ischemic brain by means of perfusion-related parameters derived from perfusion CT. Stroke. 2001;32(2):431-7.

13. Inzitari D, Pracucci G, Saia V, et al. Cerebral Reperfusion and Clinical Outcomes after Endovascular Treatment for Acute Ischemic Stroke. Neurology. 2013;16:1):1-15.

14. Zhang Y, Li C, Zhang H, Li W, Li J, Wang X. Color-coded digital subtraction angiography for assessing acute skeletal muscle ischemia reperfusion injury in a rabbit model. Acad Radiol. 2018;25(12):1609-16.

15. Miles KA, Hayball M, Dixon AK. Colour perfusion imaging: a new application of computed tomography. Lancet. 1991;337(8742):643-5.

16. Konstas AA, Wintermark $\mathrm{M}$, Lev $\mathrm{MH}$. CT perfusion imaging in acute stroke. Neuroimag Clin N Am. 2011;21(2):215-38. 
17. Guo CW, Shen SD, Zhang Y, Yi XL, Liang C, Luo W. Perfusion computed tomography evaluation of partial hepatic ischemia reperfusion in a rabbit model. Acad Radiol. 2011;18(10):1311-7.

18. Cheon JE. Quantitative Digital Subtraction Angiography in Pediatric Moyamoya Disease. J Korean Neurosurg S. 2015;57(6):432.

19. Widgerow AD. Ischemia-reperfusion injury: influencing the microcirculatory and cellular environment. Ann Plast Surg. 2014;72(2):253-60.

20. Adeva M, González-Lucán M, Seco M, et al. Enzymes involved in I-lactate metabolism in humans. Mitochondrion. 2013;13(6):615-29.

21. Carmo-Araujo EM, Dal-Pai-Silva M, Dal-Pai V, et al. Ischemia and reperfusion effects on skeletal muscle tissue: morphological and histochemical studies. Int J Exp Pathol. 2007;88(3):147-54.

22. Gawel S, Wardas M, Niedworok E, et al. Malondialdehyde (MDA) as a lipid peroxidation marker. Wiadomosci lekarskie (Warsaw, Poland: 1960). 2004; 57(9-10):453-5.

23. Bosco G, Yang Z, Nandi J, et al. Effects of hyperbaric oxygen on glucose, lactate, glycerol and anti-oxidant enzymes in the skeletal muscle of rats during ischemia and reperfusion[J]. Clinical and experimental pharmacology and physiology. 2007;34(1-2):70-6.

24. Zhang X, Zhuang Z, Ye H, et al. Objective Assessment of Transcatheter Arterial Chemoembolization Angiographic Endpoints: Preliminary Study of Quantitative Digital Subtraction Angiography. J Vasc Interv Radiol. 2013; 24(5):667-71.

\section{Publisher's Note}

Springer Nature remains neutral with regard to jurisdictional claims in published maps and institutional affiliations.

Ready to submit your research? Choose BMC and benefit from:

- fast, convenient online submission

- thorough peer review by experienced researchers in your field

- rapid publication on acceptance

- support for research data, including large and complex data types

- gold Open Access which fosters wider collaboration and increased citations

- maximum visibility for your research: over $100 \mathrm{M}$ website views per year

At $\mathrm{BMC}$, research is always in progress.

Learn more biomedcentral.com/submissions 